\title{
Fabrication of infrared-responsive carbon nanotube coating on glass surface through covalent bond formation using photoreactive silane coupling agent
}

\author{
Tomoya TAKADA ${ }^{1, \dagger}$, Yuichiro KITAMURA ${ }^{1}$ and Seisyu TAKAKUWA ${ }^{1}$ \\ ${ }^{1}$ Department of Applied Chemistry and Bioscience, Faculty of Science and Technology, Chitose Institute of Science and Technology, \\ Bibi, Chitose, Hokkaido 066-8655, Japan
}

\begin{abstract}
A carbon nanotube (CNT) layer was fabricated on a glass surface through covalent bond formation between the CNT and a photoreactive silane layer that was constructed on the glass. The silane layer contains a chlorobenzyl group $\left(-\mathrm{C}_{6} \mathrm{H}_{4}-\mathrm{CH}_{2} \mathrm{Cl}\right)$ and generates benzyl radicals upon ultraviolet light irradiation; the generated radicals smoothly attack the surface of the CNT to form covalent bonds. The X-ray photoelectron spectroscopy (XPS) and Raman spectroscopy results revealed the generation of benzyl radicals followed by the formation of covalent bonds between the glass surface and CNT. The as-formed covalent bonds improved the adhesiveness of the CNT layer on the glass surface; further, it was observed that some heat was generated upon infrared light irradiation. (c)2020 The Ceramic Society of Japan. All rights reserved.
\end{abstract}

Key-words : Carbon nanotubes, Glass, Silane coupling agent, Benzyl radical, Photothermal conversion

[Received July 8, 2020; Accepted October 2, 2020]

\section{Introduction}

Since the discovery of carbon nanotubes (CNTs) by Iijima, ${ }^{1)}$ a number of hybrid materials containing CNTs have been produced. CNTs are allotropes of carbon, consisting of cylindrically arranged condensed hexagonal rings. CNTs exhibit various industrially attractive physical characteristics such as electrical conductivity and thermal conductivity. For example, CNTs have been used as components of organic electronic devices. ${ }^{2)-9)}$ CNTs are also used as molecular heaters because they generate heat upon application of electric current ${ }^{10)-15)}$ or upon infrared light irradiation. $^{16)}$

One of the major applications of CNTs is in the surface coating of inorganic and organic materials. Until now, many researchers have reported the surface coating of various types of substrates using CNTs; the coating processes were carried out via different techniques, for example, splay coating, spin coating, bar coating, inkjet printing, and vacuum filtration. ${ }^{17)-25 \text { ) }}$

Many of the techniques introduced above are classified as physical coating; in these methods, CNTs are directly deposited on the substrate surface rather than through chemical bond formation. However, the linkage of CNTs with the substrate surface through chemical bonds is expected to be a useful method for the fabrication of durable

Corresponding author: T. Takada; E-mail: t-takada@ photon.chitose.ac.jp
CNT coatings. The chemical bond formation between CNTs and various substrates has been studied. Particularly, the chemical immobilization of CNTs on a glass surface is important for the fabrication of novel transparent functional materials. Further, the CNT-coated glass can be used as a sheet heater. The authors have studied the immobilization of CNTs on glass surfaces by means of a chemical reaction between the surface functional groups on the glass surface and CNTs. ${ }^{26)-30)}$ Tijjani et al. also studied nanocomposite fabrication using CNTs and quartz. ${ }^{31)}$

In order to modify the glass surface, functionalization with a silane coupling agent has been frequently employed. In this treatment using a silane coupling agent, chemical bonds are formed which link the glass surface to the other materials. The surface modification of the glass surface using alkoxysilane is achieved by the typical procedure shown in Fig. 1.

Hydrolysis of the alkoxy group gives a silanol group. This reaction is carried out using diluted weak acids such as acetic acid. Thereafter, the hydroxysilane generated through hydrolysis attaches to the glass surface through interaction between the silanol group on the surface and hydroxysilane. Finally, the thermal treatment of the glass results in the formation of covalent bonds between the surface and silane layer. The silane layer consists of functional groups, such as, epoxy, vinyl, and amino groups on its outer side. By means of conventional organic reactions, the functional groups of the silane layer can bind to the other organic substances. 


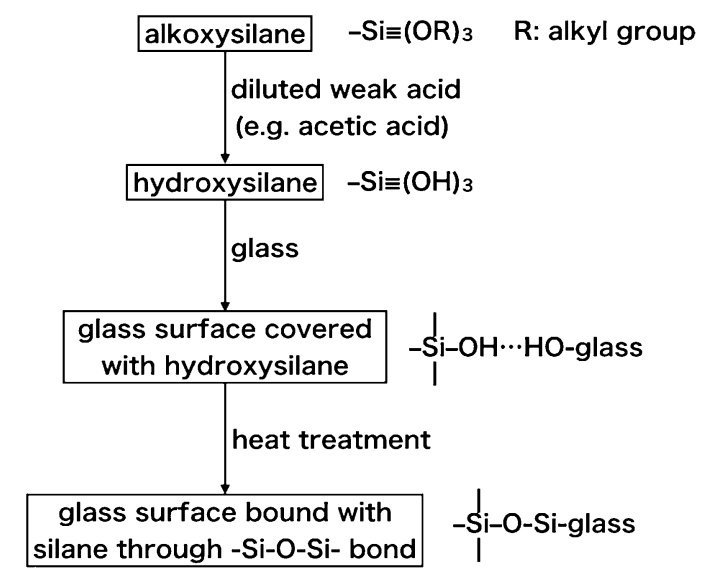

Fig. 1. Typical procedure of surface modification of glass using alkoxysilane.

In this study, we investigated the chemical immobilization of multi-walled CNTs (MWCNTs) on a glass surface using a photoreactive silane coupling agent. Although CNTs can be bound to the silane layer through various chemical processes, in order to obtain the MWCNT-coated surface via a low-cost simple modification process, novel techniques are being searched for. Therefore, the use of photo-induced reactions is promising; the reactions can proceed under mild operating conditions such as the use of less hazardous chemicals, ambient temperature, and pressure. Recently, we reported the photo-induced modification of MWCNTs with polymer side chains containing photo-dissociative moieties. ${ }^{32), 33)}$ We used poly ( $p$-chloromethylstyrene) and polyvinyl ( $p$-chloromethyl)benzoate as the photoreactive polymers. The chlorobenzyl group $\left(-\mathrm{C}_{6} \mathrm{H}_{4}-\mathrm{CH}_{2} \mathrm{Cl}\right)$ cleavages into a benzyl-type side chain radical $\left(-\mathrm{C}_{6} \mathrm{H}_{4}-\mathrm{CH}_{2} \bullet\right)$ and a chlorine atom $(\bullet \mathrm{Cl})$ upon ultraviolet light irradiation. The formation of the benzyl-type radical is known to occur through photoexcitation. ${ }^{34)}$ Most organic radicals, including benzyl-type radicals, attach to the CNT surfaces very easily. ${ }^{35)-38)}$

Although the photo-induced radicals can be applied to a glass surface coated with CNTs, the surface coating using silane coupling agents consisting of photo-dissociative moieties have not been extensively studied yet. In this work, $p$-(chloromethyl) phenyltrimethoxysilane ( $p$-CMPTMS) was used as a photoreactive silane coupling agent that was hydrolyzed with acetic acid and coated onto the glass surface. The resultant coated glass was heated in a furnace to form a covalently bound silane layer. Thereafter, the MWCNT dispersion was coated onto the glass surface and irradiated with ultraviolet light to induce benzyl-type radical formation, followed by radical addition to the MWCNT surface. This process is illustrated in Fig. 2. The chemical bond formation between MWCNTs and the silane layer was examined by an X-ray photoelectron spectroscopy (XPS) and the Raman spectroscopy. The carbon and chlorine contents in the samples were measured by the XPS. The structural changes of MWCNTs before and after the ultraviolet irradiation were observed by the Raman spectroscopy. To examine the adhesiveness of the formed

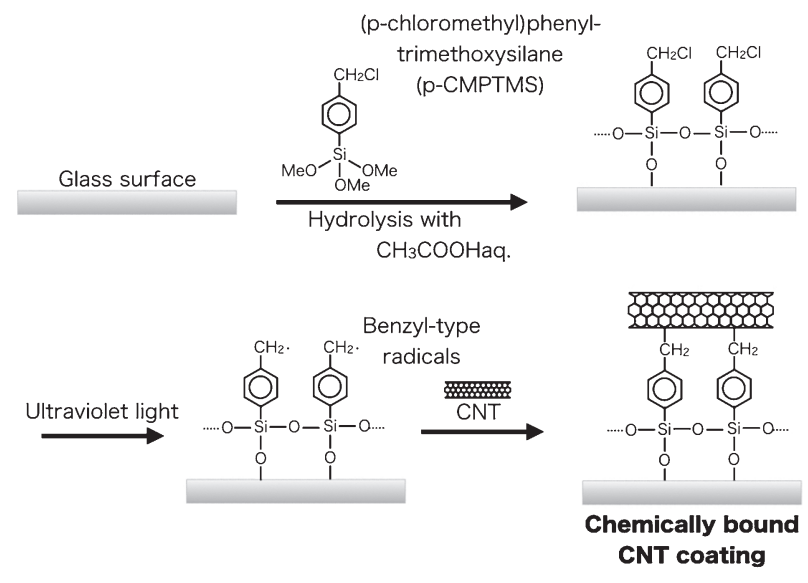

Fig. 2. Process of CNT coating using $p$-CMPTMS.

MWCNT layer, scotch-tape peeling tests and visible light transmittance measurements were performed. In addition, photothermal conversion of the fabricated MWCNT layer was also observed.

\section{Experimental procedure}

\subsection{Materials}

The MWCNTs $(10-30 \mathrm{~nm}$ in diameter, $1-10 \mu \mathrm{m}$ in length) were purchased from Wako, Japan. The carbon impurities and metal residues contained in the MWCNTs were removed by heating in a muffle furnace, followed by immersion in hydrochloric acid. Thereafter, the MWCNTs were dried in air. The soda-lime glass slips (Product ID: S1226, $76 \mathrm{~mm}$ in length, $26 \mathrm{~mm}$ in width, and $1 \mathrm{~mm}$ in thickness) were purchased from Matsunami Glass Ind., Japan. The glass slips were irradiated with vacuum ultraviolet light (wavelength: $172 \mathrm{~nm}$ ) in air to remove the residual contaminants on the surface by ozone oxidation and thereafter each of the slips were cut into three pieces. The $p$-CMPTMS was purchased from Thermo Fisher Scientific, USA. The solvents used for sample preparation (methanol and $\mathrm{N}$-methylpyrrolidone), acetic acid used as hydrolysis agent, and hydroxylammonium chloride used as dispersant ${ }^{39)}$ were purchased from Wako, Japan and used without further purification.

\subsection{Silane coating of glass surface}

Firstly, $p$-CMPTMS (1 g) was dissolved in methanol $(35 \mathrm{~mL})$ where it was hydrolyzed by dropwise addition of $1 \%$ aqueous acetic acid $(15 \mathrm{~mL})$, followed by stirring for $90 \mathrm{~min}$ in ambient temperature. The methoxy groups $\left(-\mathrm{OCH}_{3}\right)$ converted to hydroxyl groups $(-\mathrm{OH})$ during hydrolysis. The glass pieces were then put into the solution for $1 \mathrm{~h}$ by stirring, thereafter washed with methanol and dried at $65^{\circ} \mathrm{C}$ in air. After drying, the glass pieces were heated at $150^{\circ} \mathrm{C}$ for $1 \mathrm{~h}$ in a muffle furnace. The heat treatment resulted in $-\mathrm{Si}-\mathrm{O}-\mathrm{Si}-$ bond formation between the glass surface and the hydrolyzed $p$-CMPTMS. The treated glass pieces were washed with methanol again and dried at $65^{\circ} \mathrm{C}$ in air. 


\subsection{Photochemical immobilization of MWCNTs on the silane-coated glass surface}

The MWCNTs $(0.1 \mathrm{~g})$ were mixed with water $(95 \mathrm{~mL})$ and hydroxylammonium chloride $(5.0 \mathrm{~g})$ and stored for $24 \mathrm{~h}$. Thereafter, the mixture was sonicated with a bath sonicator and centrifuged for 3 min (rotating speed: 1200 rpm). The supernatant containing MWCNTs was collected and filtered using a polytetrafluorethylene membrane filter (pore size: $0.1 \mu \mathrm{m}$ ). The filtered MWCNTs were dispersed using hydroxylammonium chloride. The filter was dried at $65^{\circ} \mathrm{C}$ until water was completely vaporized. The dried filter was then placed in water and sonicated for $30 \mathrm{~min}$ to exfoliate MWCNTs from the filter. An aqueous dispersion of MWCNT was obtained as a result. The amount of MWCNTs contained in the dispersion was determined from the difference in the weight of the filter before and after the exfoliation of MWCNTs.

The aqueous dispersion was placed on the silane-coated glass surface and irradiated with ultraviolet light from an ultrahigh-pressure mercury light source (REX-250, Asahi Spectra, Japan) for $24 \mathrm{~h}$. The wavelength of the ultraviolet light was set to be longer than $300 \mathrm{~nm}$, based on the ultraviolet absorbance of $p$-CMPTMS. The MWCNT layer was formed on the surface after $24 \mathrm{~h}$ when water was completely vaporized. For the purpose of comparison, the MWCNT layer formed by vaporization of water without irradiation was also prepared.

\subsection{Characterization of the samples}

The elemental analysis of the glass surfaces was carried out using an XPS spectrometer (JPS-9200, JEOL, Japan) equipped in the Laboratory of XPS Analysis, Hokkaido University, Japan. The $\mathrm{MgK}_{\alpha} \mathrm{X}$-rays were used for the analysis. Firstly, the wide scan spectra were recorded in the range of $0-1000-\mathrm{eV}$ electron binding energy. The peaks of $\mathrm{C} 1 \mathrm{~s}$ and $\mathrm{Cl} 2 \mathrm{p}$ electrons were then recorded by a narrow scan (number of scans for integration: 10), and a background subtraction was carried out prior to the quantification of $\mathrm{C}$ and $\mathrm{Cl}$. The composition of the elements was calculated based on the peak area.

The structural changes in the MWCNTs were observed using a Raman spectrometer (InVia, Renishaw, UK). The excitation laser wavelength was $532 \mathrm{~nm}$. The bands arose from structural defects in the MWCNTs (D-band, around $1300 \mathrm{~cm}^{-1}$ ) and their original graphitic structure (G-band, around $1600 \mathrm{~cm}^{-1}$ ). The G/D ratio was calculated from the peak height.

The silane-coated glass surfaces with and without MWCNTs were observed by scanning electron microscopy (SEM) (VE-8800, KEYENCE, Japan).

The visible light transmittance of the samples was measured using a UV-Vis spectrophotometer (V-630, JASCO, Japan). The transmittance at $550 \mathrm{~nm}$ was measured before and after adhesion of a scotch-tape piece, followed by repeated peeling thrice. The scotch-tape peeling test was carried out according to the method prescribed by JIS $\mathrm{K} 5600,{ }^{40)}$ but the measurement procedure was modified to simplify the operation. The tape used was Nichiban CT, which conformed to the standard. A piece of tape was adhered to the MWCNT surface coating, and immediately peeled off. The cross-cutting of the MWCNT coating, as defined in the standard, was not performed. Instead, the visible transmittance of the peeled part was measured as mentioned above to quantify the adhesiveness of the MWCNT coating.

The temperature changes in the samples induced by the infrared light irradiation were also observed. The Infrared irradiation (wavelength: $750-1010 \mathrm{~nm}$ ) was carried out using a xenon light source (MAX-303, Asahi Spectra, Japan) and the temperature change was observed using a thermography camera (C2, FLIR, USA).

\section{Results and discussion}

\subsection{Surface observation and elemental anal- ysis of silane-coated glasses treated with MWCNTs \\ Following the reaction between $p$-CMPTMS and} MWCNT, MWCNT layers were formed on the glass surface. Figure 3 shows the SEM images of the silane-coated glass with and without MWCNT coating. A small granular deposit of silane condensate can be observed in Fig. 3(a); the silane-coated surface was not completely smooth. After MWCNT coating, the deposition of MWCNT aggregates was confirmed [Fig. 3(b)]. It was found that although MWCNTs were dispersed in water using hydroxylammonium chloride in prior, the resultant dispersion still contained aggregates.

In order to check the change in elemental composition resulting from the treatment, XPS analyses of the silanecoated glasses before and after the ultraviolet irradiation in the presence of MWCNTs were performed. Figure 4 shows the XPS spectral profiles of the silane-coated glasses treated with MWCNTs before and after the irradiation. The quantitative analysis results of $\mathrm{C}$ and $\mathrm{Cl}$ based on narrow-scan XPS are listed in Table 1.

Here, the compositions of $\mathrm{C}$ and $\mathrm{Cl}$ were calculated based on the peak area of the spectra. Only $\mathrm{C}$ and $\mathrm{Cl}$ were considered in the calculation; the sum of atomic percent of $\mathrm{C}$ and $\mathrm{Cl}$ was set at $100 \%$. The compositions of $\mathrm{Si}$ and $\mathrm{O}$ of the silanol moieties were excluded.

The $\mathrm{Cl}$ content of the glass significantly decreased when the glass was irradiated in the presence of MWCNTs. This indicates the occurrence of $\mathrm{C}-\mathrm{Cl}$ scission. The resultant radicals are added to the MWCNT surface where the silane layer is covered with MWCNT particles. Before the irradiation, the $\mathrm{C}: \mathrm{Cl}$ molar ratio of the surface chlorobenzyl groups was 87.5:12.5 (in \%); the $\mathrm{C}: \mathrm{Cl}$ ratio shown in Table 1 reflects this composition (Here the $\mathrm{C}$ content includes that of MWCNTs). After irradiation, $\mathrm{Cl}$ eliminated the benzyl radicals that were added to the MWCNTs. Therefore, only $\mathrm{Cl}$ atoms decreased in this reaction. The molar ratio shown in Table 1 is consistent with this chemical change. It is known that the $\mathrm{C} 1 \mathrm{~s}$ peak observed by XPS usually includes a signal generated from organic contaminants. Thus, a highly accurate assay of $\mathrm{C}$ based on 
(a)

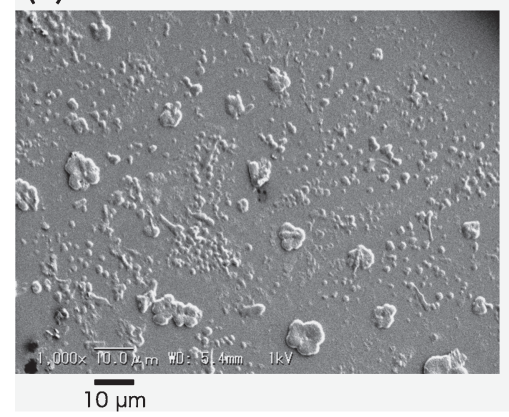

(b)

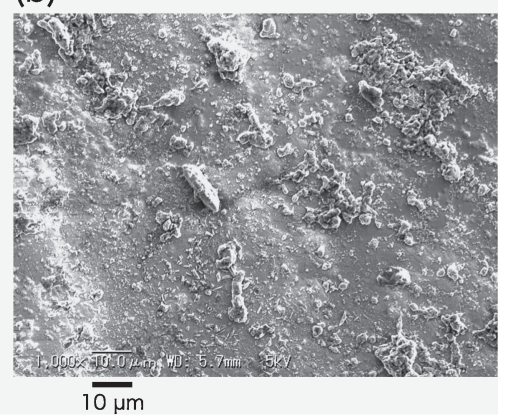

Fig. 3. SEM images of the silane-coated glasses (a) without and (b) with MWCNT coating.
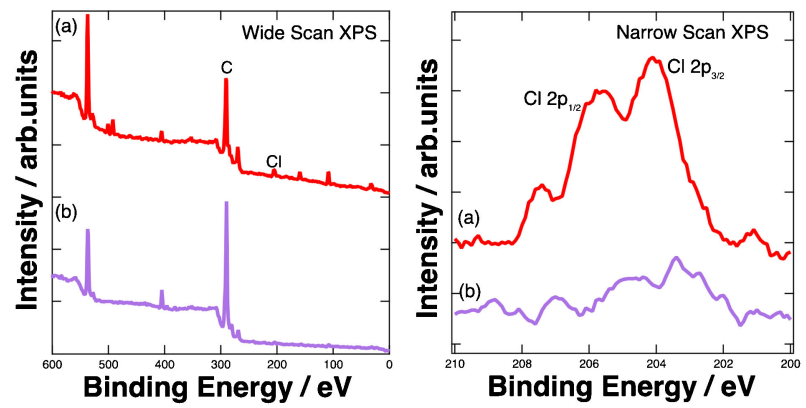

Fig. 4. Wide- and $\mathrm{Cl} 2 \mathrm{p}$ narrow-scan XPS spectral profiles of silane-coated glass (a) before and (b) after the UV irradiation in the presence of MWCNTs.

Table 1. Elemental analysis results of samples

\begin{tabular}{ccc}
\hline Sample & $\mathrm{C} / \mathrm{mol} \%$ & $\mathrm{Cl} / \mathrm{mol} \%$ \\
\hline Before Covalent Bond Formation & 96 & 4 \\
After Covalent Bond Formation & 99 & 1 \\
\hline
\end{tabular}

XPS is difficult to develop. Consequently, the elemental composition values listed in Table 1 are estimates. Although the calculated composition was not highly accurate, the decrease in $\mathrm{Cl}$ can be observed in Fig. 4; the primary purpose of the XPS experiments in this study was to observe the decrease in $\mathrm{Cl}$.

In this case, the recombination of the radicals and $\mathrm{Cl}$ atoms is a minor process, while radical addition to MWCNT particles is predominant. This is due to the difference in the density of the reaction site owing to recombination and radical addition. When the silane-coated glass is irradiated in the presence of MWCNTs, the number of carbon atoms contained in MWCNTs that are capable of bond formation is much larger than the number of $\mathrm{Cl}$ atoms generated through $\mathrm{C}-\mathrm{Cl}$ scission; therefore, the probability of radical addition to MWCNTs is higher than that of the $\mathrm{C}-\mathrm{Cl}$ recombination. The resultant free $\mathrm{Cl}$ atoms would form $\mathrm{Cl}_{2}$ molecules and escape from the reaction system because the $\mathrm{Cl}$ atom is unstable in this state.

\subsection{Structural changes of MWCNTs before and after radical addition}

When MWCNTs are attacked by radicals, defect sites are generated on the MWCNT surface. This defect forma-
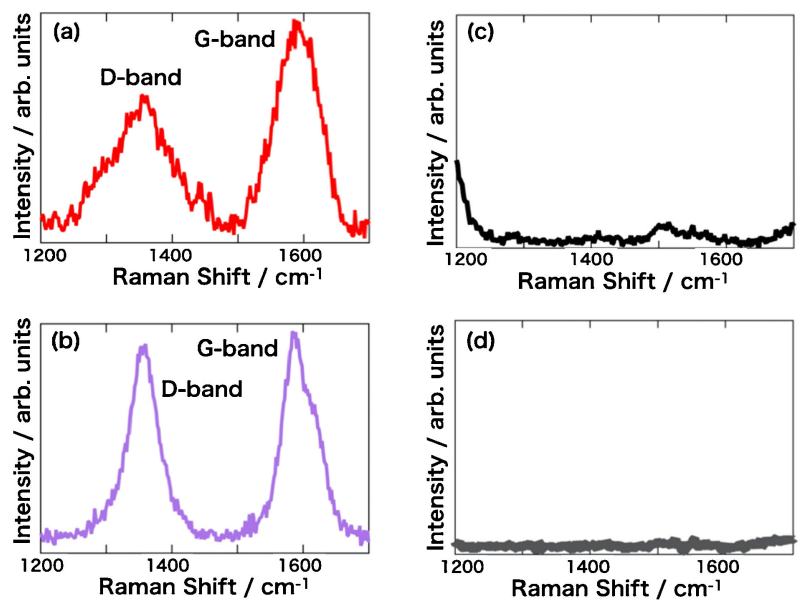

Fig. 5. Raman spectra of silane-coated glass (a) before and (b) after the UV irradiation in presence of MWCNTs. The spectral profiles of (c) intact glass and (d) silane-coated glass without MWCNT coating are also shown for comparison.

tion can be confirmed by Raman spectroscopy. Figure 5 shows the Raman spectra of the silane-coated glasses treated with MWCNTs before and after irradiation. The Gand D-bands of the MWCNTs appear in the spectra. The G-band is observed around $1600 \mathrm{~cm}^{-1}$ and is assigned to the in-plane vibrational mode of the MWCNTs. The Dband is observed around $1300 \mathrm{~cm}^{-1}$ and is assigned to the vibrational mode of the damaged sites of the $s p^{2}$-based carbon skeleton. The spectral profiles of intact and silanecoated glasses without MWCNT coating are also shown for comparison. No distinct peaks at approximately 1300 and $1600 \mathrm{~cm}^{-1}$ were observed, indicating that the peaks represented in Figs. 5(a) and 5(b) correspond to the D- and G-bands of MWCNTs.

In this study, the intensity ratio of the G- and D-bands called G/D ratio, can be used as a measure of the progress of radical addition to MWCNTs because radical addition causes structural defects in MWCNTs. The G/D ratios calculated for the samples before and after the irradiation were 1.6 and 1.1 , respectively. This result indicated that the structural defects increased with radicals. The radicals generated through the irradiation attacked and got added to the surface of MWCNTs. Taking into account the XPS and Raman results, it can be concluded that the silane layer and 


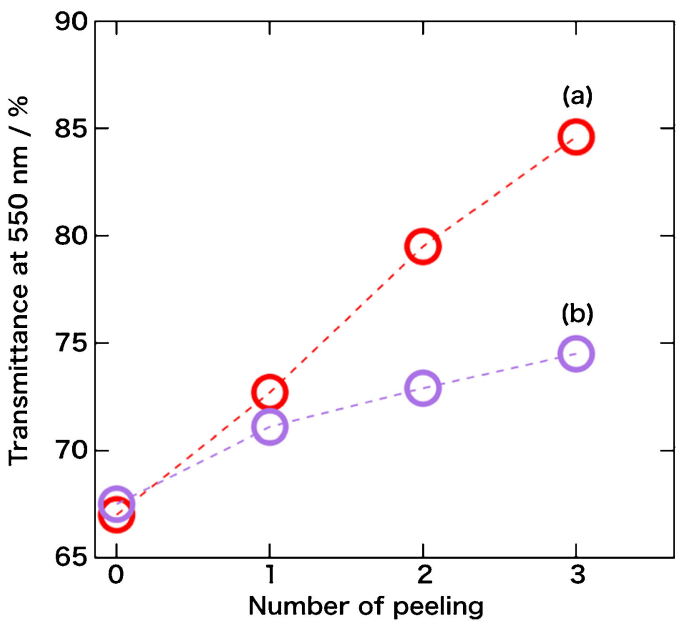

Fig. 6. Visible light transmittance of silane-coated glass (a) before and (b) after the UV irradiation in presence of MWCNTs. These transmittances were measured at a wavelength of $550 \mathrm{~nm}$.

MWCNTs are bound to each other through chemical bonds resulting from the irradiation.

\subsection{Adhesiveness of MWCNT layer before and after radical addition}

When MWCNTs are bound to the glass surface through chemical bonds, the MWCNT layer firmly adheres to the surface compared with the layer formed without chemical bonds. To compare the adhesiveness of the MWCNT layer fabricated with and without the ultraviolet irradiation, scotch-tape peeling tests were performed. Figure 6 shows the visible light transmittance as a function of the number of peeling.

The results clearly indicate the suppression of detachment of the MWCNT layer prepared by the irradiation. Transmittance is a measure of the amount of MWCNTs remaining on the glass; a higher transmittance value corresponds to a smaller amount of MWCNTs, and vice versa. The visible transmittance value of the MWCNT layer immediately after fabrication is approximately $67 \%$. This value is common to all the samples prepared with and without irradiation. On peeling with a scotch tape, the differences in the visible transmittance values change distinctly.

The sample prepared without the irradiation showed a significantly increased transmittance value. This indicates that the MWCNT layer easily detaches from the glass surface. Conversely, the rise in the transmittance value observed in the sample prepared with the irradiation was relatively small compared with that in the sample prepared without the irradiation; the MWCNT layer detached from the surface slightly. The MWCNT aggregates observed in Fig. 3(b) were detached by peeling, but covalently bound MWCNTs were considered to be firmly immobilized on the surface. On the basis of these results, we can conclude that the adhesiveness of the MWCNT layer can be improved by the photo-induced chemical bond formation.

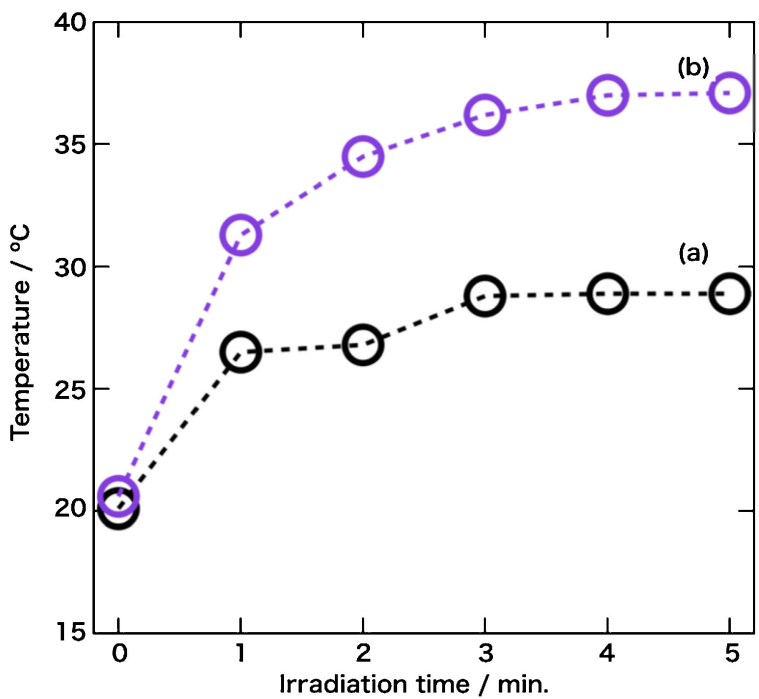

Fig. 7. Temperature change observed for (a) intact glass and (b) silane-coated glass covered with a covalently bound MWCNT layer.

\subsection{Photothermal conversion characteristics of the prepared materials}

MWCNTs are known to effectively convert infrared radiation energy to heat energy. ${ }^{16)}$ This photothermal conversion can be observed through temperature measurements. In this study, the photothermal conversion characteristics of the MWCNT layer fabricated on a glass surface were evaluated. Figure 7 shows the temperature change observed in the silane-coated glass irradiated with ultraviolet light in the presence of MWCNTs as a function of the infrared irradiation time. The temperature change of an intact glass is also shown for the purpose of comparison. The initial temperatures of these samples were almost equal. Upon the infrared irradiation, the silane-coated glass covered with MWCNTs showed a significant temperature increase. This result indicated that the photothermal conversion capacity of MWCNTs remained unchanged despite the immobilization on the glass surface through chemical bond formation. In contrast, the intact glass showed a relatively minor temperature increase. Therefore, the significant temperature rise noted above is mainly due to the photothermal conversion caused by the MWCNTs. Based on the observed characteristics, the materials fabricated by the present method are expected to be used as transparent sheet heaters in the future.

\section{Summary}

The fabrication of an MWCNT layer on a glass surface through the addition of benzyl radicals generated by the photo-dissociative silane coupling agent, $p$-CMPTMS, was carried out to create an infrared-responsive exothermic coating. The XPS, Raman, and visible transmittance observations revealed that the chemical bond formation between the surface silane layer and MWCNTs was successfully achieved through photo-induced benzyl radical formation, followed by addition of the resultant radicals to the MWCNT surface. The fabricated MWCNT layer showed 
infrared-induced heat generation. Therefore, the MWCNTcoated glass has various applications, such as a selfheating transparent material for the defrosting windows of the vehicles used in cold regions and a panel of measuring instruments operated in a low-temperature environment. One of the important topics that remain to be studied further is the dependence of the infrared-induced temperature change on the amount of MWCNTs immobilized. It has been reported that in polyvinyl ( $p$-chloromethyl)benzoate/MWCNT composites, the infrared-induced temperature change depends on the MWCNT content. ${ }^{33)} \mathrm{A}$ similar tendency will be observed in the case of MWCNTcoated glasses. Another pending subject is the effect of the glass composition on adhesiveness. If the composition varies, the yield of the silane coating and the adhesiveness of the MWCNT layer may be influenced. The effect of the difference in composition on the adhesiveness should be further examined.

Acknowledgments Some parts of this study were partially supported by the Suzuki Foundation and Takahashi Industrial and Economic Research Foundation. This work was technically supported by the Nanotechnology Platform Program (Synthesis and Materials, Microstructure Characterization) of the Ministry of Education, Culture, Sports, Science and Technology (MEXT), Japan. The X-ray photoelectron spectra were recorded using the equipment installed in the Laboratory of XPS analysis (technical staff: Mr. Keita Suzuki), Hokkaido University. Further, we would like to thank Editage (www.editage.jp) for English language editing.

\section{References}

1) S. Iijima, Nature, 354, 56-58 (1991).

2) M. Kaempgen, G. S. Duesberg and S. Roth, Appl. Surf. Sci., 252, 425-429 (2005).

3) G. Hu, C. Zhao, S. Zhang, M. Yang and M. Wang, Polymer, 47, 480-488 (2006).

4) D. O. Kim, M. H. Lee, J. H. Lee, T.-W. Lee, K. J. Kim, Y. K. Lee, T. Kim, H. R. Choi, J. C. Koo and J.-D. Nam, Org. Electron., 9, 1-13 (2008).

5) H. J. Park, J. Kim, J. Y. Chang and P. Therato, Langmuir, 24, 10467-10473 (2008).

6) E. Logakis, P. Pissis, D. Pospiech, A. Korwitz, B. Krause, U. Reuter and P. Pötschke, Eur. Polym. J., 46, 928-936 (2010).

7) E. Logakis, C. Pandis, P. Pissis, J. Pionteck and P. Pötschke, Compos. Sci. Technol., 71, 854-862 (2011).

8) L. Zhao, W. L. Liu, L. D. Zhang, J. S. Yao, W. H. Xu, X. Q. Wang and Y. Z. Wu, Colloid. Surface. A, 423, 69$76(2013)$.

9) W.-Y. Lin, Y.-F. Shih, C.-H. Lin, C.-C. Lee and Y.-H. Yu, J. Taiwan Inst. Chem. E., 44, 489-496 (2016).

10) S. Isaji, Y. Bin and M. Matsuo, Polymer, 50, 1046-1053 (2009).

11) Z. P. Wu and J. N. Wang, Physica E, 42, 77-81 (2009).

12) T. J. Kang, T. Kim, S. M. Seo, Y. J. Park and Y. H. Kim, Carbon, 49, 1087-1093 (2011).

13) D. Jung, D. Kim, K. H. Lee, L. J. Overzet and G. S. Lee,
Sens. Actuators, A, 199, 176-180 (2013).

14) D. Janas and K. K. Koziol, Carbon, 59, 457-463 (2013).

15) D. Kim, L. Zhu, D.-J. Jeong, K. Chun, Y.-Y. Bang, S.-R. Kim, J.-H. Kim and S.-K. Oh, Carbon, 63, 530-536 (2013).

16) N. W. S. Kam, M. O'Conell, J. A. Wisdom and H. Dai, P. Natl. Acad. Sci. USA, 102, 11600-11605 (2005).

17) C. M. Trottier, P. Glatkowski, P. Wallis and J. Luo, J. Soc. Inf. Display, 13, 759-763 (2005).

18) J. T. Han, S. Y. Kim, H. J. Jeong and G.-W. Lee, Ind. Eng. Chem. Res., 48, 6303-6307 (2009).

19) Y. Zhou, L. Hu and G. Grüner, Appl. Phys. Lett., 88, 123106 (2006).

20) Z. Wu, Z. Chen, X. Du, J. M. Logan, J. Sippel, M. Nikolou, K. Kamaras, J. R. Reynolds, D. B. Tanner, A. F. Hebard and A. G. Rinzler, Science, 27, 1273-1276 (2004).

21) A. A. Green and M. C. Hersam, Nano Lett., 8, 14171422 (2008).

22) Y. Wang and B. Fugetsu, Carbon, 82, 152-160 (2015).

23) B. Dan, G. C. Irvin and M. Pasquali, ACS Nano, 3, 835843 (2009).

24) J.-W. Song, J. Kim, Y.-H. Yoon, B.-S. Choi, J.-H. Kim and C.-S. Han, Nanotechnology, 19, 095752 (2008).

25) Y.-I. Lee, S. Kim, K.-J. Lee, N. V. Myung and Y.-H. Choa, Thin Solid Films, 536, 160-165 (2013).

26) S. Abe, K. Nakayama, D. Hayashi, T. Akasaka, M. Uo, F. Watari and T. Takada, Phys. Proc., 14, 147-151 (2011).

27) S. Abe, K. Nakayama, H. Kobayashi, T. Kiba, T. Akasaka, S. Sato, M. Uo, F. Watari and T. Takada, Nano Biomed., 3, 208-216 (2011).

28) K. Nakayama, T. Takada, S. Abe, Y. Honda, H. Ikeyama, Y. Nakaya and A. Furusaki, Mol. Cryst. Liq. Cryst., 568, 38-45 (2012).

29) T. Takada, Y. Konno, K. Nakayama, P. T. Dunuwila, Y. Maeda and S. Abe, Nano Biomed., 4, 113-117 (2012).

30) Y. Konno, T. Takada and S. Abe, TANSO, 266, 7-10 (2015).

31) Y. Tijjani, F. M. Yasin, M. H. S. Ismail and A. H. M. Ariff, J. Ceram. Soc. Japan, 126, 984-991 (2018).

32) T. Takada, T. Baba and S. Abe, C, 2, 20 (2016).

33) T. Takada, Y. Nishioka and T. Baba, $C, 3,28$ (2017).

34) G. Porter and F. J. Wright, Trans. Faraday Soc., 51, 1469-1474 (1951).

35) H. Peng, L. B. Alemany, J. L. Margrave and V. N. Khabashesku, J. Am. Chem. Soc., 125, 15174-15182 (2003).

36) N. Tsubokawa, Polym. J., 37, 637-655 (2005).

37) I. Fenoglio, M. Tomatis, D. Lison, J. Muller, A. Fonseca, J. B. Nagy and B. Fubini, Free Radic. Biol. Med., 40, 1227-1233 (2006).

38) A. Galano, J. Phys. Chem. C, 112, 8922-8927 (2008).

39) Y. Sabba and E. L. Thomas, Macromolecules, 37, 48154820 (2004).

40) Japanese Industrial Standards, "Testing methods for paints - Part 5: Mechanical property of film - Section 6: Adhesion test (Cross-cut test)", K5600-5-6 (1999) [in Japanese]. 